\title{
Morphological Characteristics of Unfertilized Eggs of the Japanese Sardine, Compared with Fertilized Ones*1
}

\author{
Masanobu Matsuoka and Yoshinobu Konishi \\ Seikai National Fisheries Research Institute, Kokubu, Nagasaki 850, Japan \\ (Received December 13, 1995)
}

\begin{abstract}
Morphological changes of unfertilized and fertilized eggs of the Japanese sardine Sardinops melanostictus were observed continuously. Unfertilized sardine eggs could be divided into three types on the basis of their morphology: a distorted type, a narrow perivitelline-space type, and a normal perivitelline-space type. The first type of eggs disintegrated and sank to the bottom of the containers in a short time. The latter two types of eggs formed not only a perivitelline space but also a blastodisc. They disintegrated gradually within 12 hours after stripping. Common to eggs of these three types, the egg membrane finally broke and the egg's contents were mainly lost. The examination of the field-collected eggs showed that some wild eggs were very similar in their morphological characteristics to unfertilized and disintegrated sardine eggs mentioned above. These facts suggest that unfertilized sardine eggs may be commonly present in natural spawning.
\end{abstract}

Key words: Japanese sardine, Sardinops melanostictus, unfertilized egg, fertilized egg, morphological characteristics, disintegration, wild egg

The Japanese sardine Sardinops melanostictus is one of the most important commercial fishes in Japan for food, feeds for fish culture, and so forth. The annual catch by weight of this species was the highest among all fishes in Japan through the 1980s. However, the annual catch has been rapidly declining since 1989 , raising concerns about the stock abundance. To clarify the mechanisms underlying the fluctuations in sardine population size, detailed information about their reproductive biology ${ }^{1)}$ and developmental biology ${ }^{2}$ is needed.

Matsuoka et $a l .{ }^{3)}$ made a successful artificial fertilization of Japanese sardine eggs and described the subsequent embryonic development. In their observations, some eggs formed a perivitelline space and a blastodisc, but did not develop further. It was doubtful whether such eggs were actually fertilized, because the eggs of some teleostean fishes such as salmonids and cyprinids can be activated simply by contact with water. ${ }^{4,5)}$ Relevant here, Nakai') distinguished fertilized sardine eggs from unfertilized ones, based simply on their diameters and widths of the perivitelline space. With this criteria, he examined sardine eggs collected with nets and suggested that in natural spawnings nearly $100 \%$ were fertile. More precise information is thus required on the morphology of the "unfertilized egg" of the Japanese sardine. This information may then be used to estimate total fertilized egg productions by the spawning survey method. ${ }^{\text {? }}$

The present study was undertaken to clarify the morphological changes with time of both unfertilized and fertilized eggs of the Japanese sardine. Based on this result, the possibility of the presence of unfertilized sardine eggs occurring in natural spawning was discussed.

\section{Materials and Methods}

Adult sardines were caught by gill net in the Amakusanada waters $\left(32^{\circ} 26^{\prime} \mathrm{N}, 129^{\circ} 30^{\prime} \mathrm{E}\right)$ during a cruise of the $\mathrm{R} / \mathrm{V}$ Yoko Maru. The gill net (length, $50 \mathrm{~m}$; height, $10 \mathrm{~m}$; mesh size, $48 \mathrm{~mm}$ ) was lowered to a depth of $35 \sim 40 \mathrm{~m}$ from 20:00 to 20:30, on March 13 and 14, 1993. The sampling depth and time accorded with their spawning time ${ }^{* 2,8)}$ and depth. ${ }^{* 3}$ A total of 383 specimens including fully ripe females and males were caught for the fertilization experiments.

Five experiments were made, using 4 ripe females with hydrated eggs and 4 ripe males, 3-4 hours after capture. In each experiment, ca. 1,000 of the stripped eggs from a female were divided into two groups, and one group of eggs was directly placed into sea water (non-inseminated group) and the other group was inseminated by spermatic fluid stripped from a male, using the "dry method" (inseminated group). The eggs were maintained in petri dishes of $15 \mathrm{~cm}$ diameter or in $1 /$ beakers, and incubated at $17.2 \sim 17.8^{\circ} \mathrm{C}$. Some eggs were removed at intervals and observed and photographed under the microscope, then fixed in $10 \%$ formalin-sea water solution. These fixed eggs were used for reexamination in the laboratory after the cruise.

Field-collected eggs were examined to assess the possibility of occurrence of unfertilized eggs in the natural spawning ground. These were collected by horizontal hauls at five layers of plankton nets $(60 \mathrm{~cm}$ diameter and with General Oceanics Double Trip Mechanism as the net closing apparatus) at a location $\left(30^{\circ} 55^{\prime} \mathrm{N}, 130^{\circ} 29^{\prime} \mathrm{E}\right)$, on March 2, 1992.

\footnotetext{
* Contribution from Seikai National Fisheries Research Institute, No.521.

$*_{2}$ Abst. Metg. Japan. Soc. Fisheries Sci., October, 1991, p. 32 (in Japanese).

${ }^{*}$ Abst. Metg. Japan. Soc. Fisheries Sci., October, 1993, p. 107 (in Japanese).
} 


\section{Results}

Sardine eggs immediately after stripping from the female were almost spherical in shape and floated on the sea water surface. No conspicuous perivitelline space was visible in them (Fig. 1A). It was noticed that some eggs were slightly distorted and not spherical in shape. Within about 20 minutes after stripping from the female, these anomalous eggs sank to the bottom of the containers and their inner structure showed marked disintegration (Fig. 1B). At fifteen minutes after stripping, some normally shaped eggs of both the non-inseminated and inseminated groups began to form narrow, distinct perivitelline spaces (Fig. 1C). At about 30 minutes, the perivitelline space expanded outward and the blastodisc was formed at the animal pole in both the non-inseminated (Fig. 1D) and inseminated eggs.
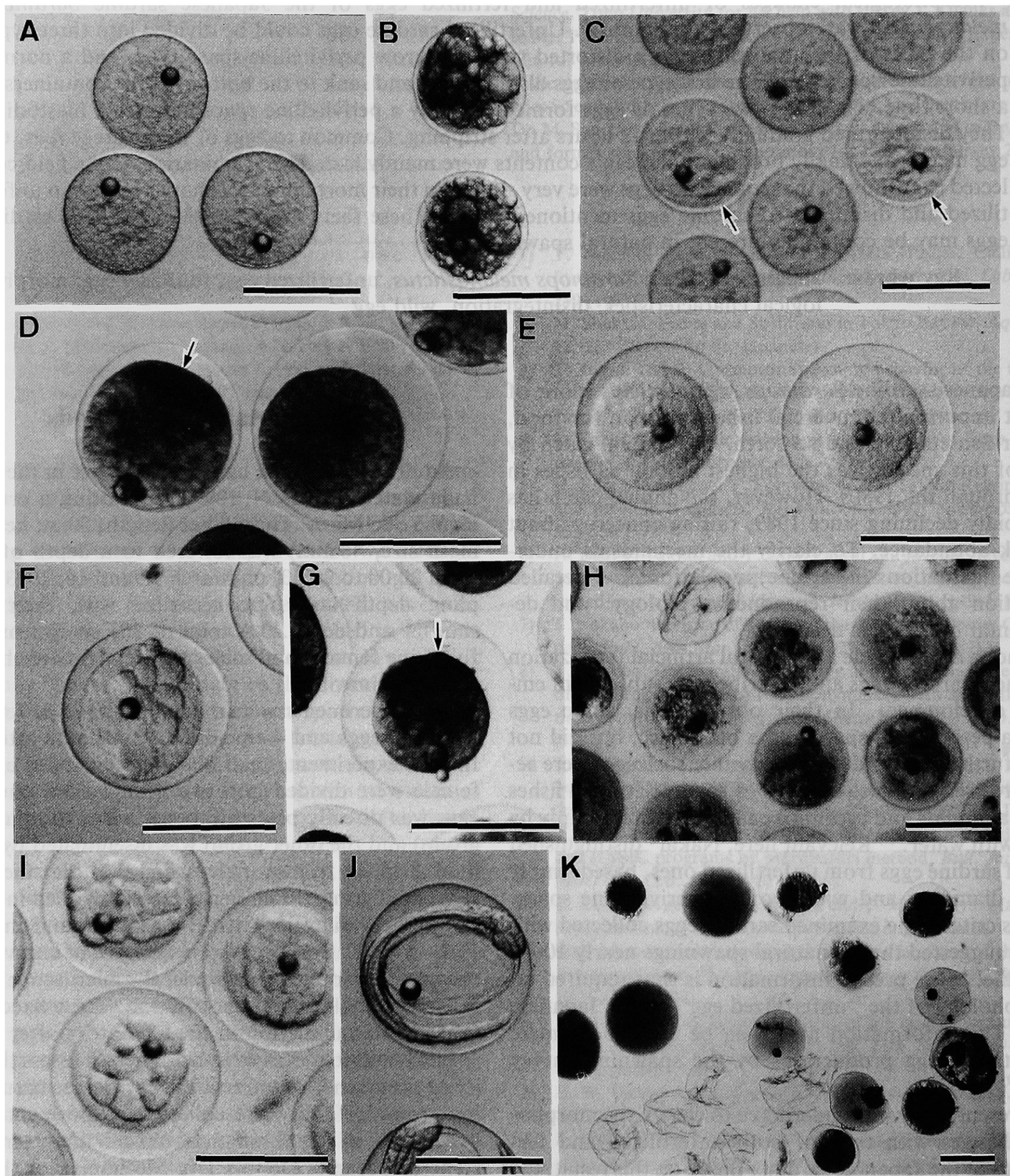

Fig. 1. Unfertilized (A-H, K) and artificially fertilized (I, J) eggs of the Japanese sardine.

A: immediately after stripping out. B: distorted and disintegrated eggs ( $20 \mathrm{~min}$ after stripping out). C: initial formation of the perivitelline space (arrows) (15 min). D: initial formation of blastodisc (arrow) (32 min, formalin-fixed eggs). E: formation of fully developed perivitelline space $(1.15 \mathrm{~h})$. F: irregularly cleft surface of blastodisc $(1.65 \mathrm{~h})$. G: slightly roughened surface (arrow) of blastodisc ( $3.25 \mathrm{~h}$, formalin-fixed egg). $\mathrm{H}$ : disintegrated and sunk eggs with cloudy appearance $(11.73 \mathrm{~h})$. I: 8 and 16-cell stage eggs $(1.58 \mathrm{~h})$. J: C stage) egg (40.67 h). K: various types and stages of disintegrated eggs $(11.58 \mathrm{~h})$. Scale bars indicate $1 \mathrm{~mm}$. 
Figure 1E shows the non-inseminated eggs in which a fully developed perivitelline space and distinct blastodiscs were formed at 1.15 hours after stripping. There were also some eggs which had a narrower perivitelline space and a less distinct blastodisc than those in Fig. 1E.

The blastodisc of non-inseminated eggs subsequently exhibited a curiously rugged appearance beyond 1.5 hours after stripping, including irregularly shaped cleft cells or bubbles (Fig. 1F). This rugged appearance was lost in the eggs preserved in formalin, although a slightly roughened surface was observed (Fig. 1G). Further observation confirmed that the rugged blastodisc and yolk of non-inseminated eggs disintegrated shortly afterwards and the eggs sank to the bottom of the containers within 12 hours after stripping. The blastodisc and yolk fragmented in the egg, so the entire egg showed a cloudy appearance (Fig. 1H).

Fertilized eggs underwent normal cleavage, embryo formation (Fig. 1I, J) and hatching, as described by Matsuoka et al. ${ }^{3)}$

Figure $1 \mathrm{~K}$ shows the eggs which were inseminated but not fertilized and then disintegrated. They all sank to the bottom of the containers. Although the degree of disintegration varied in appearance, a similar process occurred in such unfertilized eggs as in the non-inseminated ones. At the final stage of disintegration, the egg membrane was broken and the contents leaked out.

Progressive changes of the morphology and structure of unfertilized (Fig. 2, 1-13) and fertilized (Fig. 2, 14-18) sardine eggs are diagramatically summarized in Fig. 2. Three types of unfertilized eggs were distinguished in all ex- periments: a distorted type (Fig. 2, 1-3), a narrow perivitelline-space type (Fig. 2, 4-8), and a normal perivitellinespace type (Fig. 2, 9-13). The normal pervitelline-space type (Fig. 2,10) of unfertilized eggs was the same in appearance as the fertilized eggs (Fig. 2, 15) until the cleavage stage.

Examination of wild eggs collected by the plankton net revealed that some eggs exhibited similar morphological features to the unfertilized and disintegrated sardine eggs described above. For example, Fig. 3A shows disintegrated eggs having little contents except for the oil globule and Fig. 3B shows eggs which disintegrated yet still had their contents. These disintegrated eggs were caught at $40 \mathrm{~m}$ depth by a horizontal haul and occupied $55 \%$ in a sample of the total numbers of sardine eggs including normal ones. The vertical distributions of disintegrated anomalous eggs and normal ones are shown in Fig. 4. Considerably fewer anomalous eggs were collected at about 1 , 20,60 , and $80 \mathrm{~m}$ depth than at $40 \mathrm{~m}$ depth.

\section{Discussion}

According to Miller, ${ }^{9}$ unfertilized eggs of the Pacific sardine Sardinops caerulea formed no perivitelline space when incubated for three hours in sea water. Addition of sperm after the incubation resulted in successful fertilization of only one egg, in which the perivitelline space was subsequently formed. In contrast, our observations on the Japanese sardine $S$. melanostictus confirmed that the unfertilized eggs could be activated by contact with sea water

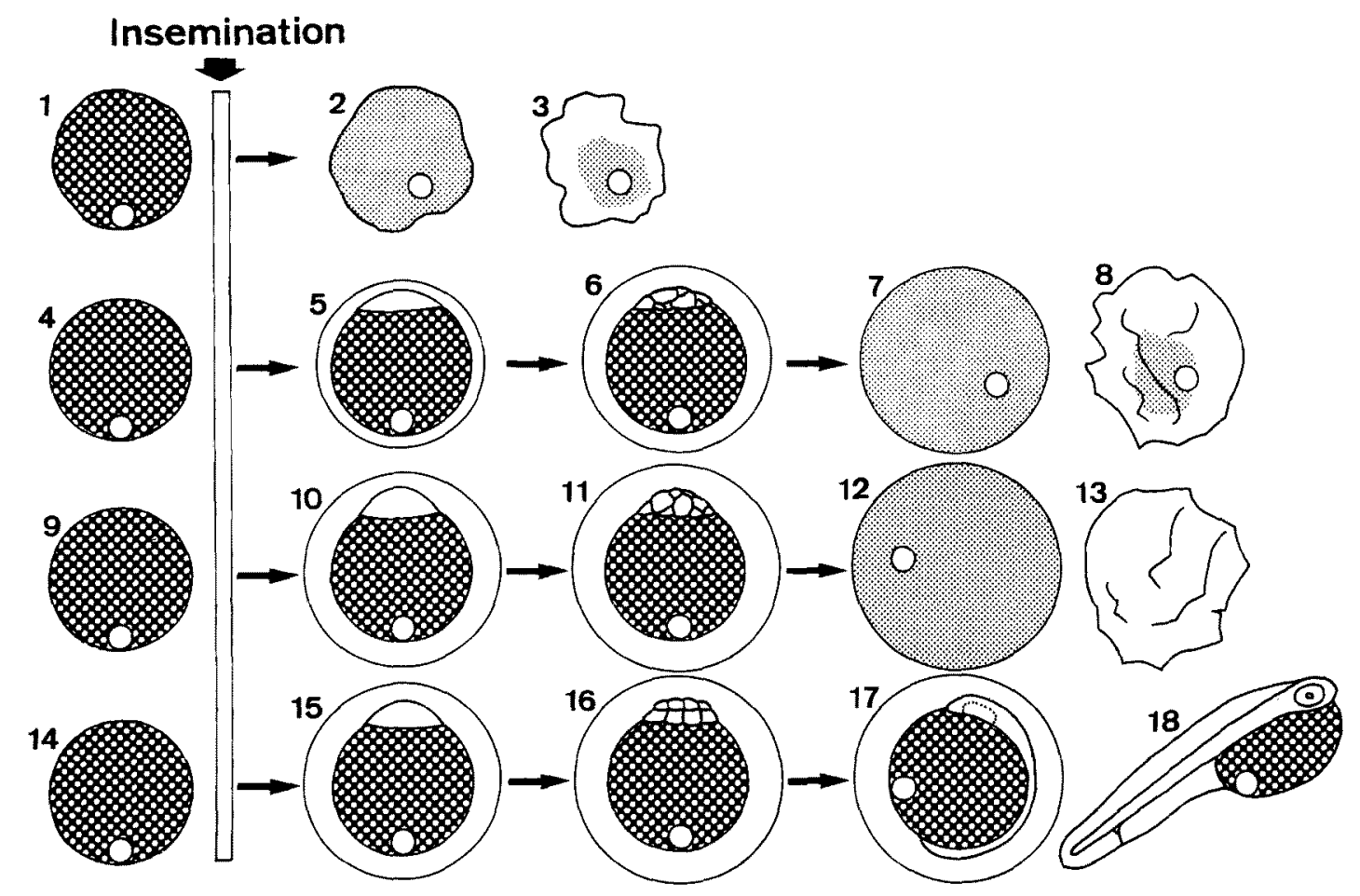

Fig. 2. Schematic illustrations of morphological changes with time in three types of unfertilized eggs (1-13) and artificially fertilized eggs (14-18) of the Japanese sardine.

1-3: unfertilized (distorted type). 4-8: unfertilized (narrow perivitelline-space type). 9-13: unfertilized (normal perivitelline-space type). 14-18: fertilized and developing. 


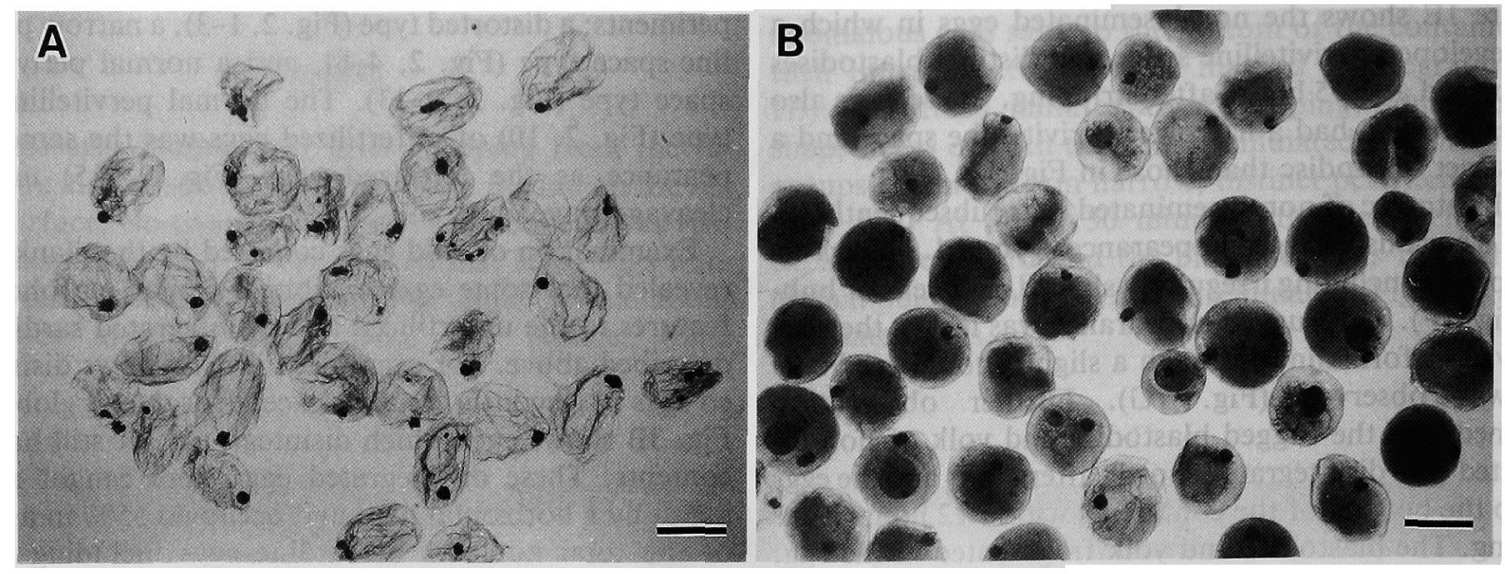

Fig. 3. Anomalous wild eggs of the Japanese sardine collected in natural spawning area.

A: disintegrated eggs having little contents except for the oil globule. B: eggs which disintegrate yet still have their contents. Scale bars indicate $1 \mathrm{~mm}$.

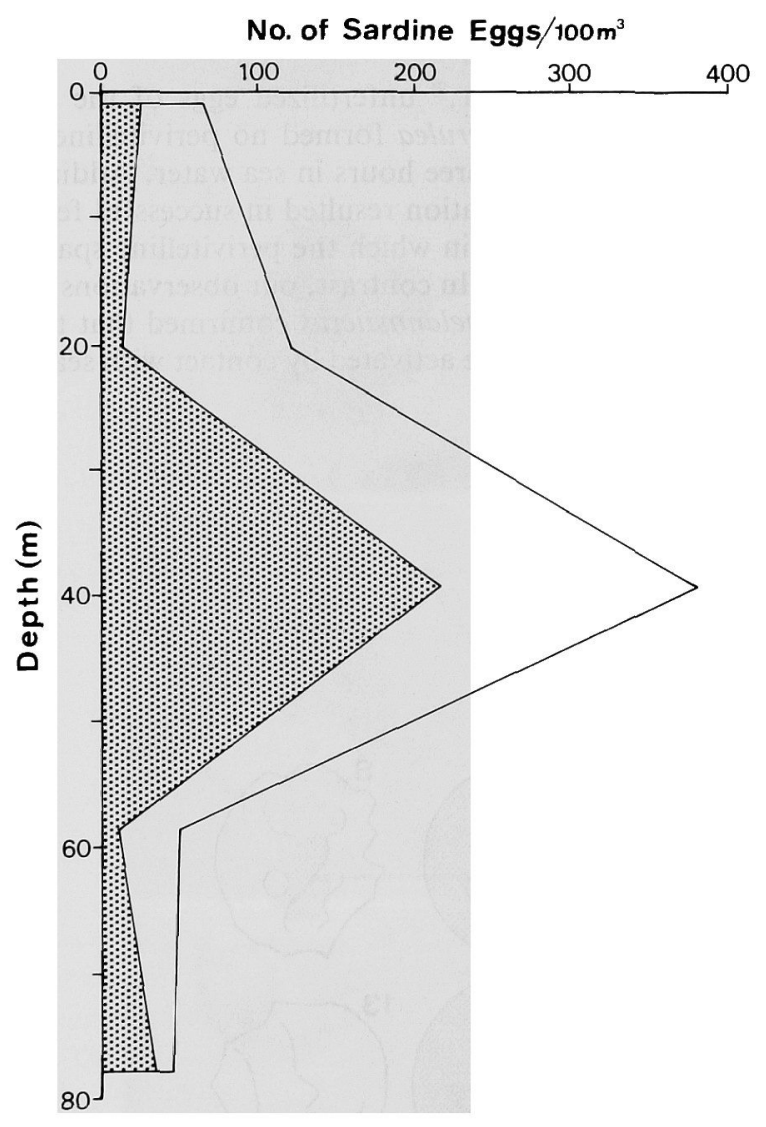

Fig. 4. Vertical distribution of disintegrated anomalous wild eggs and normal ones of the Japanese sardine.

Stippled area, anomalous eggs. Open area, normal eggs.

and form a conspicuous perivitelline space and blastodisc without the addition of sperm as found in salmonids and cyprinids. ${ }^{4,5)}$ In the case of the Pacific sardine, ${ }^{9)}$ it was unclear whether the observed formation of the perivitelline space was entirely due to fertilization or not. Nagahama ${ }^{4}$ pointed out that successful fertilization in fish eggs should be confirmed by using the cleavage as an index.

Three types of progressive changes of unfertilized eggs were observed in this study. The occurrence of these three types may be due to slight differences in the maturation condition of eggs. These types were found in both the noninseminated and inseminated groups, because the fertilization success in the inseminated group was very low in the present experiments. Delayed artificial fertilization, 3-4 hours after capture of adult sardine, may cause a low success rate of fertilization, although $\mathrm{Nakai}^{6}$ reported that the fertilization ability of eggs and sperm was maintained for 5-6 hours after capture in the present species.

With regard to the wild egg of the Japanese sardine, Nakai $^{6}$ identified only 7 unfertilized eggs out of 395,910 eggs in plankton net samples from a total of 676 stations during the period of 1936 to 1943 . He considered only spherical eggs with no or very narrow perivitelline space as unfertilized ones. From this result, he regarded that nearly $100 \%$ of sardine eggs were fertilized in natural spawning. It may be that there were few unfertilized eggs in the samples caught by the surface hauls which Nakai') used, because unfertilized and disintegrated sardine eggs have a tendency to sink. The vertical distribution of disintegrated anomalous wild eggs showed that there were few eggs at the surface layer (Fig. 4). From the present study, it is now clear that there are several types of unfertilized eggs and that they change morphologically with time after release in sea water. The distorted and disintegrated sardine eggs collected at $40 \mathrm{~m}$ depth in the natural spawning area shown in Fig. 3A, B are morphologically very similar to the disintegrated, unfertilized eggs of Fig. 1K and Fig. 2. Although Nishimura ${ }^{10)}$ suggested that sardine eggs from the cleavage stage to the closure of blastopore are apt to be damaged by mechanical stimuli such as net sampling, these eggs of Fig. $3 \mathrm{~A}, \mathrm{~B}$ are unlikely to be simply damaged by such stimuli since they do not include any cleft cells and the condition of disintegration of egg content and membrane is variable. Burst egg membranes and eggs with a cloudy appearance similar to those of Fig. 1K and Fig. 2 have been also often collected by spawning surveys. Therefore, it may be appropriate to consider that most of the disintegrated sardine eggs shown in Fig. 3A, B are examples of unferti- 
lized eggs, and that unfertilized sardine eggs are usually present in natural spawning, in contrast to Nakai's view.

One important problem for estimating total egg productions by the spawning survey method may be the fact that the normal perivitelline-space type of unfertilized sardine eggs can not be distinguished from fertilized eggs prior to the cleavage stage. This means that some unfertilized eggs may be included in total egg productions. In this respect, further examination and sampling in deeper layers and comprehensive areas will be required for quantitative estimations of unfertilized sardine eggs in the sea.

Recent advances in artificial induction of spawning and larval fish rearing have revealed that egg quality is of primary importance in larval survival. Kjørsvik et al. ${ }^{11}$ demonstrated that chromosome aberrations in developing eggs was a reliable criteria for poor-quality eggs of seacaught Atlantic cod Gadus morhua with less survival potential. In the Japanese sardine, some chemical components that are essential for early development of hydrated eggs collected from the sea-caught female are variable depending on sampling groups. ${ }^{12)}$ These findings suggest that there are considerable numbers of non-viable eggs in the sea even in eggs naturally spawned, presumably depending on maternal nutritional conditions. Presumptive unfertilized sardine eggs found in the sea may be due to poorquality eggs which have little potential for successful development as well as those which fail to encounter sperm. In order to understand the possible mechanisms for the fluctuations in the Japanese sardine stock, further study on the quality of eggs spawned in the sea is required.

Acknowledgments We wish to express our thanks to Professor Dr. Masaru Tanaka of Kyoto University, Dr. Tsutomu Ikeda of Nansei National Fisheries Research Institute (present, Professor of Hokkaido University), and Dr. Chris Norman, STA fellow (present, Chiba University), for their critical reading of the manuscript.

\section{References}

1) M. Matsuyama, T. Fukuda, S. Ikeura, Y. Nagahama, and S. Matsuura: Spawning characteristics and steroid hormone profiles in the wild female Japanese sardine Sardinops melanostictus. Fisheries Sci., 60, 703-706 (1994).

2) M. Matsuoka and T. Mitani: Hatching and rearing experiments on eggs of Japanese sardine, Sardinops melanostictus, collected from adjoining area of the Nagasaki Harbor (Preliminary study). Bull. Seikai Reg. Fish. Res. Lab., 67, 15-22 (1989)(in Japanese).

3) M. Matsuoka, K. Miyaji, and O. Katoh: Artificial fertilization and egg development of the Japanese sardine. Bull. Seikai Natl. Fish. Res. Inst., 73, 45-51 (1995) (in Japanese).

4) Y. Nagahama: Reproduction: regulatory mechanisms of gametogenesis, in "Fish Physiology" (ed. by Y. Itazawa and I. Hanyu), Koseisha-Koseikaku, Tokyo, 1991, pp. 243-286 (in Japanese).

5) R. Suzuki: Developmental physiology and biotechnology, in "Fish Physiology" (ed. by Y. Itazawa and I. Hanyu), Koseisha-Koseikaku, Tokyo, 1991, pp. 585-609 (in Japanese).

6) Z. Nakai: Studies of infuences of environmental factors upon fertilization and development of the Japanese sardine eggs-with some reference to the number of their ova. Bull. Tokai Reg. Fish. Res. Lab., 9, 109-150 (1962).

7) T. Watanabe: Spawning survey method, in "Population Dynamics of Fishery Resources" (ed. by T. Ishii), Koseisha-Koseikaku, Tokyo, 1983, pp. 9-29 (in Japanese).

8) H. Morimoto: Time of maximal oocyte hydration and spawning in the Japanese sardine in Tosa Bay, southwestern Japan. Nippon Suisan Gakkaishi, 59, 7-14 (1993) (in Japanese).

9) D. J. Miller: Development through the prolarval stage of artificially fertilized eggs of the Pacific sardine (Sardinops caerulea). Calif. Fish and Game, 38, 587-595 (1952).

10) S. Nishimura: Occurrence of the abnormal sardine eggs in the plankton sample obtained by net hauling, with some ecological notes thereupon. Ann. Rept. Jap. Sea Reg. Fish. Res. Lab., 4, 87 103 (1958) (in Japanese).

11) E. Kjфrsvik, A. Mangor-Jensen, and I. Holmefjord: Egg quality in fishes. Adv. Mar. Biol., 26, 71-113 (1990).

12) H. Morimoto: Effects of maternal nuturitional conditions on number, size, and lipid content of hydrated eggs in the Japanese sardine from Tosa Bay, southwestern Japan, in "Survival Strategies in Early Life Stages of Marine Resources" (ed. by Y. Watanabe, Y. Yamashita, and Y. Oozeki), Balkema, Rotterdam, 1996, pp. 3-12. 